\section{Vigencia de la semiótica}

Desiderio Blanco

\section{Objeto de la semiótica}

uando en una conversación cual-
quiera digo que enseño semiótica, la primera pregunta de mi interlocutor es: ¿Y eso qué es? Y al informarle que la semiótica es una ciencia que estudia los procesos de significación, la segunda pregunta no se hace esperar: ¿Y eso $p a-$ ra qué sirve? La respuesta ahora resulta más compleja. En primer lugar, porque si sabemos qué es la semiótica, podremos decir para qué sirve.

Pues bien, la semiótica es una ciencia, o como prefería decir A. J. Greimas más modestamente, un proyecto científico que tiene por objeto de estudio la significación: cómo se produce y cómo se aprehende la significación. La significación no es algo dado de antemano; es el resultado de un proceso de producción. Y puede considerarse desde dos perspectivas: o como proceso, es decir, la significación en acto; o como producto, establecido y terminado en un texto. Cuando hablamos de "texto" no nos limitamos al texto literario, oral o escrito; una película, en ese sentido, es un texto; es un texto una pintura y una fotografía, como lo es igualmente un partido de fútbol o la procesión del Señor de los Milagros. Es "texto" todo aquello que tiene sentido.

\section{Sentido y significación}

Entre sentido y significación hay que hacer algunas distinciones. El sentido es 
ante todo una dirección. Y así hablamos de una avenida de doble sentido, de una calle de un solo sentido. Decir que "algo" tiene sentido es decir que tiende hacia alguna cosa. Esa "tensión" y esa "dirección" son constitutivas del sentido. La condición mínima para que una "materia" cualquiera produzca un efecto de sentido es que se halle sometida a una intencionalidad.

La significación, en cambio, es un producto organizado por el análisis, por ejemplo, el contenido de sentido vinculado a una expresión, una vez que esa expresión ha sido aislada y que se ha verificado que ese contenido y esa expresión se encuentran ineluctablemente vinculados. La significación está, pues, ligada a una unidad, cualquiera que sea su tamaño. La unidad óptima es sin duda el discurso. Por eso hablamos siempre de la significación de algo. En consecuencia, la significación está siempre articulada, mientras que el sentido está simplemente orientado. Dicho de otro modo, la orientación es una propiedad del sentido; la articulación es una propiedad de la significación. La articulación se efectúa por diferencias, por grados, por jerarquías, por dependencias, por polarizaciones, etcétera.

\section{Percepción y significación}

Percibir una cosa es ante todo percibir una presencia, antes incluso de reconocer su figura. En efecto, antes de identificar una figura del mundo natural, o una noción o un sentimiento cualquiera, percibimos (o "presentimos") su presencia, es decir, algo que, por una parte, ocupa cierta posición en relación con nuestra propia posición, y cierta extensión, y que, por otra parte, nos afecta con cierta intensidad. La presencia, cualidad sensible por excelencia, es una primera articulación semiótica de la percepción. El afecto que nos embarga, esa intensidad que caracteriza nuestra relación con el mundo, esa tensión en dirección al mundo, es asunto de la mira intencional. La posición, la extensión y la cantidad, caracterizan, en cambio, los límites y el contenido del dominio de pertinencia, es decir, la captación. Así, pues, la mira y la captación son las dos operaciones elementales para que la presencia comience a significar; ellas constituyen las dos modalidades que guían el flujo de la atención hacia la significación.

Pero para que un sistema de valores semióticos ${ }^{*}$ adquiera cuerpo, es preciso que surjan diferencias y que esas dife-

* Los valores semióticos, o lingüísticos, no son los valores axiológicos. Los primeros son creados por diferencias entre los elementos de un campo semiótico; son los valores saussurianos: un término lingüístico vale por su diferencia con otro u otros términos, no por su sustancia: verticalidad obtiene su valor 
rencias constituyan una red coherente. esa es la condición de lo inteligible. La significación surge siempre de un entrecruzamiento entre lo sensible y lo inteligible. Por eso, el sistema de valores semióticos resulta de la conjugación de una mira y de una captación; una mira que guía la atención hacia una primera variación, que es la intensiva, y una captación, que pone en relación esa primera variación con otra, de naturaleza extensiva, y que delimita así los contornos comunes de sus respectivos dominios de pertinencia.

La mira y la captación son operaciones elementales que realiza la instancia del cuerpo propio, definido por J. Fontanille como la forma significante de una experiencia sensible. El cuerpo propio es el órgano de la dimensión propioceptiva, desde la cual participa tanto de los fenómenos del mundo exterior -dimensión exteroceptiva- como de los fenómenos del mundo interior-dimensión interoceptiva-. La instancia del cuerpo propio se desplaza incesantemente por el campo en el que se halla instalado, o campo de pre- sencia. Con sus desplazamientos, determina, en el campo en el que toma posición, una brecha entre el universo exteroceptivo y el universo interoceptivo, entre la percepción del mundo exterior y la percepción del mundo interior, instalando entre ambos mundos las modificaciones de la frontera misma. En tal sentido, la semiosis se encuentra en perpetuo movimiento, y lo que en un momento constituía el plano del contenido, en el siguiente puede pasar a constituir el plano de la expresión de un nuevo plano del contenido. Si el cuerpo percibiente asocia el color de una fruta [plano de la expresión] con la condición de "maduro" [plano del contenido], puede desplazarse en el campo perceptivo para asociar ahora "lo maduro" [plano de la expresión] con la estación del otoño [plano del contenido], y con un nuevo desplazamiento, asociar luego estación de otoño [plano de la expresión] con la edad madura del hombre [plano del contenido].

La significación supone entonces un mundo de percepciones, donde el

por su diferencia con horizontalidad, vida por su diferencia con muerte, extenso por su diferencia con restringido, etcétera. Los valores axiológicos resultan del investimiento de los valores semióticos por la categoría tímica: euforia vs. disforia. Es así como los objetos, neutros por naturaleza, se tiñen, mejor se empapan de afectividad, y resultan objetos agradables o desagradables, bellos o feos, útiles o inútiles, buenos o malos, sagrados o profanos, etcétera. Los valores axiológicos corresponden a lo que el sentido común llama simplemente "valores"; los valores semióticos o lingüísticos pertenecen al sistema virtual de la lengua o del código, cualquiera que sea. 
cuerpo propio, al tomar posición, instala globalmente dos macrosemióticas, cuya frontera puede desplazarse siempre, pero que tiene cada una su forma específica. De un lado, la interoceptividad da lugar a una semiótica que tiene la forma de una lengua natural o de otro tipo de código, y de otro lado, la exteroceptividad da lugar a una semiótica que tiene la forma de una semiótica del mundo natural. La significación es, pues, el acto que reúne esas dos macrosemióticas, y eso es posible gracias a la instancia del cuerpo propio del sujeto de la percepción, cuerpo propio que tiene la propiedad de pertenecer simultáneamente a las dos macrosemióticas entre las cuales toma posición.

\section{Estilos de categorización}

Una de las capacidades fundadoras de la actividad de lenguaje (de todo lenguaje) es la capacidad de categorizar el mundo, de clasificar sus elementos. [La tabla de Mendeleiev es, en ese sentido, un lenguaje]. No se puede concebir un lenguaje incapaz de producir tipos, pues de lo contrario necesitaría una expresión para cada ocurrencia, lo que sería del todo inmanejable. Lo que manipulan los lenguajes, incluidos los lenguajes no-verbales, son tipos de objetos (por ejemplo, un escritorio en general) y no ocurrencias de objetos (por ejemplo, el escritorio particular que se encuentra en mi oficina). Únicamente el discurso podrá evocar, luego o para- lelamente, gracias al acto de referencia, tal o cual ocurrencia del tipo para ponerla en escena.

En el dominio de la imagen, por ejemplo, la necesidad de hacer referencia a tipos visuales se ha confundido durante largo tiempo con la necesidad de nombrar los objetos representados. La imagen de un árbol no es la imagen de ese árbol porque yo puedo llamarla "árbol", sino porque se acerca al tipo visual "árbol". Del mismo modo, si reconozco una forma redondeada elíptica, no es porque la puedo llamar "elipse", sino porque en ella reconozco el tipo visual "elipse". El que no conozca el nombre y se vea obligado a utilizar una perífrasis ["algo redondo aplastado"], no por eso dejaría de reconocer el tipo visual.

La formación de tipos es en cierta forma otro nombre de la categorización. Esa es la formación de clases que todo lenguaje manipula. E interesa a todos los órdenes del lenguaje: la percepción, el código y su sistema. Pero la categorización se pone en marcha especialmente en el discurso, puesto que preside la instalación de los "sistemas de valores".

Existen varias maneras de formar categorías de lenguaje. La manera clásica en semántica estructural ha sido la búsqueda de rasgos pertinentes, llamados semas, hasta formar el lexema. La formación de la categoría reposa en 
ese caso en la identificación de esos rasgos comunes, en su número y en la distribución de estos entre los miembros de la categoría. Se la ha denominado categorización por serie.

Una versión vaga de ese procedimiento es la manera de categorización que Wittgenstein llamó semejanza de familia. En un conjunto de parientes, las semejanzas que permiten reconocerlos están desigualmente distribuidas: los hijos se parecen al padre, que se parece a la tía, que se parece a la madre, que se parece a los hijos, etcétera. Cada semejanza difiere de la siguiente, y finalmente, no hay nada en común entre el primer elemento y el último. No obstante, la pertenencia de cada individuo al grupo no ofrece duda.

Pero se puede también organizar una categoría en torno a una ocurrencia particularmente representativa, más fácilmente identificable que todas las demás, y que posee en sí misma todas las propiedades que solo parcialmente se encuentran en cada uno de los otros miembros de la categoría. En esa manera de categorizar se basa precisamente la figura de la antonomasia. La formación de la categoría reposa ahora en el mejor ejemplar del conjunto, lo que llamamos el parangón. [El gorrión es el parangón de la clase de los pájaros].

En cambio, podemos elegir para formar la categoría la ocurrencia más neutra, aquella que solo posee algunas propiedades comunes a todas las de- más. Así, para designar los recipientes destinados a la cocción hablamos de "ollas" sin mayores determinaciones. La formación de la categoría reposa en ese caso en la elección de un término de base, neutro.

Esos cuatro estilos de categorización se basan ante todo en elecciones perceptivas, y sobre todo en la manera como se percibe y se establece la relación entre el tipo y sus ocurrencias: la categoría puede ser percibida, en extensión, como una distribución de rasgos, como una serie (unida por uno o varios rasgos comunes), o como una familia (unida por un "aire de familia"); puede, en cambio, ser percibida como la agrupación de sus miembros en torno a uno solo de ellos (o de una de sus especies), formando un agregado en torno a un término de base; o un parangón, reconocido como el "mejor ejemplar" de la categoría. Para cada una de esas elecciones, la categoría nos puede proporcionar, en base a su propia morfología, un sentimiento de unidad fuerte o débil: en el caso de la serie y del parangón, el sentimiento de unidad es fuerte; en el caso del agregado y de la familia, ese sentimiento de unidad es débil.

En suma, los "estilos de categorización" nos remiten a las dos grandes dimensiones de la "presencia", aunque ahora se trata del modo de presencia del tipo en la categoría: puede presentar una extensión difusa o concentra$\mathrm{da}$, y una intensidad sensible fuerte o débil: 


\begin{tabular}{|c|c|c|c|}
\hline & & \multicolumn{2}{|c|}{ Extensión } \\
\hline & & concentrada & difusa \\
\hline \multirow{2}{*}{ Intensidad } & fuerte & $\begin{array}{c}\text { mejor ejemplar } \\
\text { [parangón] }\end{array}$ & $\begin{array}{c}\text { rasgos comunes } \\
\text { [serie] }\end{array}$ \\
\hline & débil & $\begin{array}{c}\text { base neutra } \\
\text { [conglomerado] }\end{array}$ & $\begin{array}{c}\text { semejanza de familia } \\
\text { [familia] }\end{array}$ \\
\hline
\end{tabular}

\section{Recorrido de la significación}

Para llegar a su plena articulación, la significación sigue un recorrido inmanente a lo largo del discurso. Ese recorrido hipotético-deductivo es meramente teórico y nada tiene que ver con el proceso psíquico que tiene lugar en la mente del autor. Este es un recorrido genético, aquel es un recurrido generativo. El recorrido generativo de la significación va de los elementos más simples a los más complejos, de los más abstractos a los más concretos, de los más profundos a los más superficiales. Y lo mismo sucede con las sucesivas articulaciones del sentido.

Las primeras articulaciones de la significación, las más profundas y abstractas, son las articulaciones de las estructuras elementales. En la Semiótica clásica esas estructuras elementales se organizan en base a dos tipos de oposiciones: las oposiciones privativas [A/A] o contradictorias, y las oposiciones cualitativas $[\mathrm{A} / \mathrm{B}]$ o contrarias. El rasgo de / masculino/ que distingue, por ejemplo, al término "padre" es cualitativamente contrario al rasgo de /femenino/ que caracteriza al término "madre". Esa relación de contrariedad se articula en una estructura elemental como la siguiente:

\section{/masculino/ $\longleftrightarrow$ /femenino/}

en la que la flecha de doble dirección señala el eje común del género, y los términos /masculino/ y /femenino/ indican los polos opuestos de la categoría, como resultado de la relación de contrariedad. Las oposiciones privativas se expresan como negaciones de los términos contrarios:

$$
\begin{gathered}
\text { /masculino/ } \longleftrightarrow \longrightarrow \text { /no masculino/ } \\
\text { /femenino/ } \longleftrightarrow \text { /no femenino/ }
\end{gathered}
$$

Una nueva articulación entre ambas oposiciones da lugar al modelo constitucional de la significación, que se expresa por medio del conocido cuadrado semiótico, el cual constituye la piedra angular de la semiótica clásica: 
/masculino/

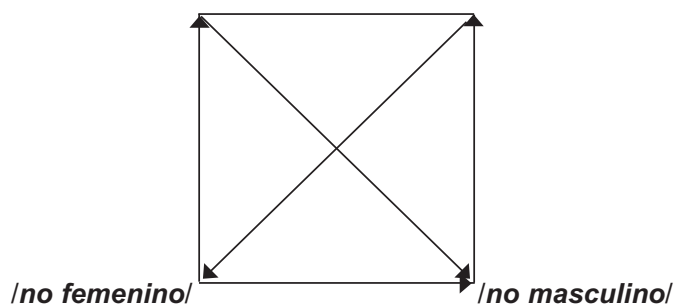

De la nueva articulación surgen dos nuevos términos contrarios, llamados por comodidad "sub-contrarios", generados en torno a un eje neutro. Pero, a su vez, las relaciones de base generan una nueva relación entre los términos / masculino/ y / no femenino/ y entre los términos /femenino/ y / no masculino/: una relación de complementariedad.

En la simplicidad del modelo reside la gran potencia explicativa que ofrece. El cuadrado semiótico representa un microuniverso de sentido, y no solamente valora los cuatro términos polares en él expresados; da cuenta también de los grados intermedios que los discursos concretos puedan actualizar. Si decimos, por ejemplo: Esa chica es poco femenina, actualizamos una posición en el cuadrado, que va de lo /femenino/ a lo /no femenino/. Si, por el contrario, decimos: Ese hombre es afeminado, nuestro discurso actualiza una posición entre lo / no masculino/ y lo /femenino/.
La más moderna semiótica tensiva pretende afinar esos grados de significación por medio de otro modelo: el esquematismo tensivo. Este dispositivo trabaja la correlación entre las dos dimensiones de la presencia sensible: la intensidad y la extensidad. A partir de esas dos dimensiones, consideradas como dimensiones graduales, su correlación puede ser representada por el conjunto de puntos de un espacio sometido a dos ejes de control:

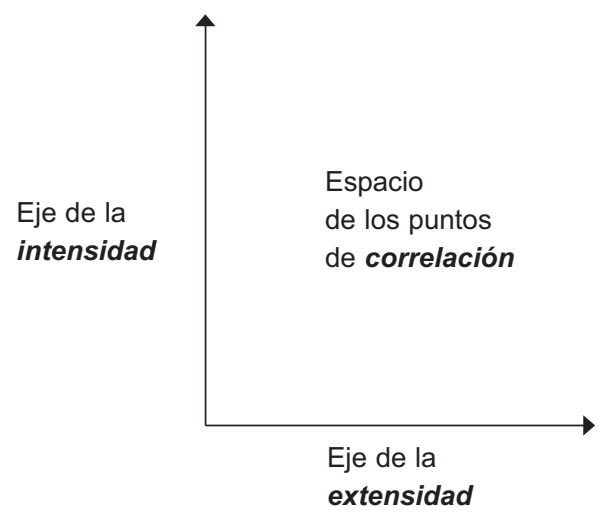

La intensidad caracteriza el dominio de lo sensible; la extensidad caracteriza el dominio de lo inteligible. La correlación entre los dos dominios resulta de la toma de posición de un "cuerpo propio", sede del efecto de la presencia sensible. La toma de posición del "cuerpo propio" impone una orientación al rumbo del sentido por medio de la "mira" [=poner en la mira], 
a partir del dominio interno, es decir, de la intensidad, y por medio de la "captación", a partir del dominio externo, o sea, de la extensidad. La "mira" y la "captación" convierten las dimensiones graduales en ejes de profundidad, orientadas a partir de una posición de observación. Los grados de la intensidad y de la extensidad, bajo el control de las operaciones de la "mira" y de la "captación" se convierten en grados de profundidad perceptiva.

Si se consideran los puntos del espacio interno de correlación, uno por uno, todas las combinaciones entre los grados de cada uno de los dos ejes son posibles, todos están disponibles para definir las diferentes posiciones del sistema. Lo importante, sin embargo, no son las posiciones aisladas, sino los valores, es decir, las posiciones relativas, las diferencias de posición.

Los dos ejes del espacio externo definen las valencias de la categoría. Todos los puntos del espacio interno son susceptibles de corresponder a $v a-$ lores de la misma categoría. Pero de esa nube de puntos se desprenden algunos principios organizadores: de un lado, la diferencia entre las dos correlaciones determina dos grandes zonas de correlación: la zona de correlación inversa y la zona de correlación conversa (o directa); del otro, la conjugación de los grados más fuertes y más débiles de los dos ejes determina zonas extremas. Todos los puntos del es- pacio interno son pertinentes, pero las zonas extremas de cada correlación son las zonas más típicas de la categoría en cuestión.

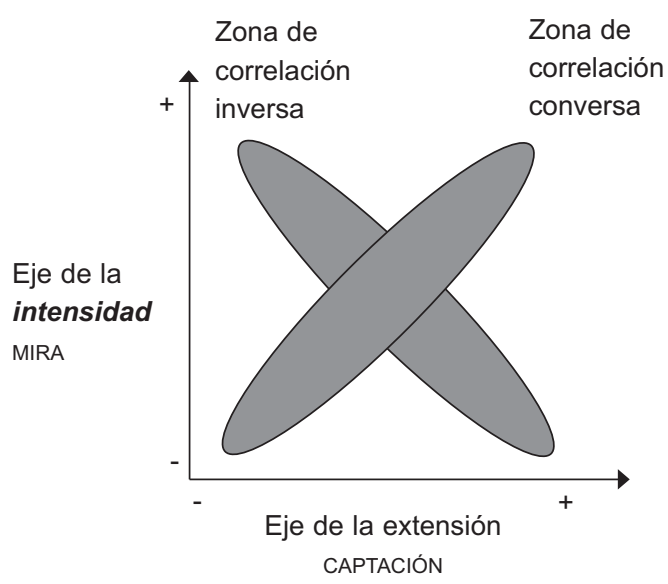

La combinación entre esos dos principios permite desprender cuatro grandes zonas típicas de la categoría, que corresponden, además, a los "estilos de categorización”, ya enumerados anteriormente:

- Una zona de intensidad fuerte y de extensión débil (o concentrada): estilo categorial: el parangón.

- Una zona de intensidad y de extensión igualmente fuertes: estilo categorial: la serie.

- Una zona de intensidad débil y de extensión fuerte (o difusa): estilo categorial: la familia.

- Una zona de intensidad y de extensión igualmente débiles: estilo categorial: el conglomerado. 


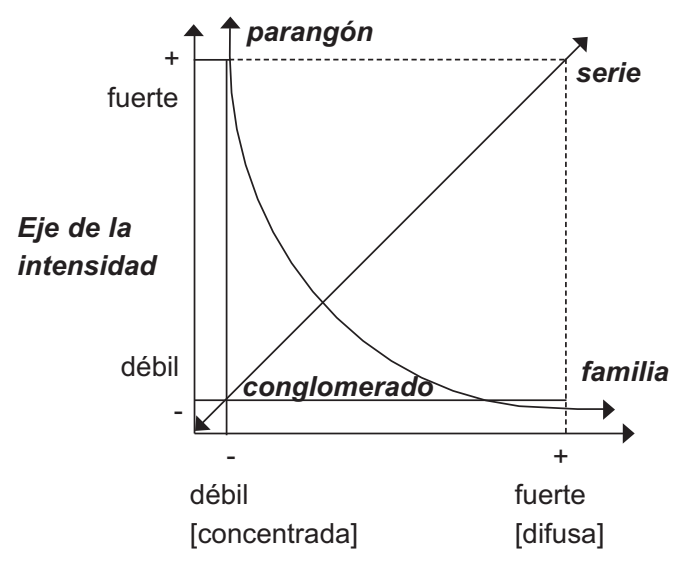

Eje de la extensión

\section{Isotopía}

El concepto de isotopía se forma bajo la inspiración de los fenómenos descritos por la físico-química. Un isótopo es un nucleido que tiene el mismo número atómico que otro, cualquiera que sea su número de masa. Todos los isótopos de un elemento tienen las mismas propiedades químicas.

En el dominio semiótico, las "mismas propiedades" semánticas surgen de la redundancia de determinados semas. Para entender ese fenómeno discursivo, es preciso aclarar que un sema es la unidad mínima de significación con la que se inicia la articulación del sentido. Los semas son rasgos distintivos de los lexemas, que no existen aisladamente, pero que nos permi- ten diferenciar los "objetos semióticos" entre sí. Así, el rasgo de /verticalidad/ que compone el lexema "columna", o el rasgo de /horizontalidad/ que integra el lexema "viga", o el rasgo de /masculinidad/ que define al lexema "padre", son semas.

Los semas son de dos clases: aquellos que constituyen el "núcleo" más o menos permanente del lexema, y aquellos otros que emergen del contexto. Los primeros son denominados semas nucleares; los segundos, semas contextuales o clasemas, porque cumplen una función clasificadora. Un ejemplo permitirá ilustrar esas operaciones: en enunciados como

- LLas columnas del Partenón son particularmente bellas]

- [A mi padre le duele la columna]

- [El Papa es la columna de la Iglesia]

el lexema "columna" manifiesta semas nucleares, o específicos, tales como

/verticalidad/, /fijeza/, /soporte//resistencia/, /articulación/, /consistencia/, entre otros;

pero en cada enunciado propuesto, la relación contextual del lexema "columna" con lexemas como "Partenón", "padre", "Iglesia", pone de manifiesto otros semas como /arquitectónico/, /anatómico/, /institucional/, propios también del lexema "columna", pero no específicos, no nucleares, porque no son necesarios para que columna 
sea "columna", aunque son requeridos para saber de qué columna se trata. El contexto interno de cada enunciado nos permite aprehender que en el primer enunciado se habla de una "Columna arquitectónica”; en el segundo, de una "columna anatómica"; en el tercero, de una "columna institucional". Como puede observarse, los semas /arquitectónico/, /anatómico/, /institucional/ permiten clasificar el lexema "columna". Por tal razón, esos semas contextuales son llamados clasemas. Gracias a ellos, podemos hablar de "columnas arquitectónicas", de "columnas anatómicas" o de "columnas institucionales". Los clasemas cumplen además otra función sumamente importante: obligan al enunciado a seleccionar del acervo virtual del lexema aquellos semas nucleares que son coherentes con el contexto del enunciado, dejando de lado aquellos otros que no lo son. El clasema/arquitectónico/ que surge del contexto del primer enunciado, selecciona los semas nucleares /verticalidad/ /fijeza/, /soporte/, /resistencia/, /consistencia/; pero no /articulación/, por ejemplo. El clasema /anatómico/ que surge del contexto del segundo enunciado, selecciona los semas nucleares/verticalidad/, /soporte/, /resistencia/, /articulación/, /consistencia/; pero no /fijeza/. El clasema/institucional/ que emerge del contexto del tercer enunciado, selecciona los semas /soporte/, /consisten- cia/, /articulación/; pero no /verticalidad/, /fijeza/ ni /resistencia/.

La articulación combinatoria de semas mucleares [Ns] y de clasemas [Cls] da por resultado una nueva entidad semiótica, denominada semema. El semema es un equivalente de la noción lingüística de "acepción". En el primer enunciado entendemos columna como "columna arquitectónica"; en el segundo enunciado, la columna se presenta como "columna anatómica"; en el tercer enunciado, la columna surge como "COlumna institucional". Cada tipo de columna que cada enunciado genera en base a una contextualización diferente es un semema: "Columna arquitectónica", "columna anatómica", "columna institucional" son sememas.

El semema es una unidad semiótica de manifestación de sentido. Es una unidad más compleja que el sema, y de un nivel jerárquicamente superior. Lo que "captamos" en la lectura, lo que "vemos" en cada visión de una película, en cada contemplación de una pintura, son siempre sememas, nunca semas ni lexemas: aquellos por ser abstractos, estos por ser virtuales.

En la construcción del semema intervienen, como acabamos de ver, semas nucleares [Ns] y clasemas [Cls]. Y existen cuatro posibilidades de combinación entre ellos: 
I

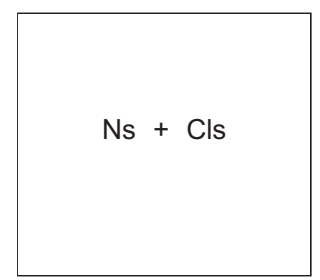

II

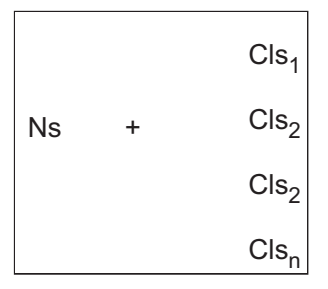

III

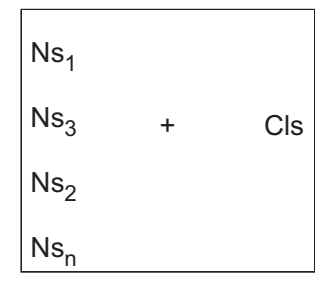

IV

\begin{tabular}{lll|}
$\mathrm{Ns}_{1}$ & & $\mathrm{Cls}_{1}$ \\
$\mathrm{Ns}_{2}$ & + & $\mathrm{Cls}_{2}$ \\
$\mathrm{Ns}_{3}$ & & $\mathrm{Cls}_{3}$ \\
$\mathrm{Ns}_{\mathrm{n}}$ & & $\mathrm{Cls}_{\mathrm{n}}$ \\
\hline
\end{tabular}

Cuatro perfiles isotópicos diferentes. Porque la isotopía consiste en la reiteración de semas a lo largo del discurso, sea la repetición de clasemas, sea la repetición de semas nucleares. Esta última repetición da lugar a la isotopía semiológica; la primera origina la isotopía semántica. En el caso (I), el discurso resultante de esa combinatoria es un discurso unisótopo: el discurso científico, el discurso filosófico, y todo discurso que trate de evitar la ambigüedad. El caso (II) da origen a los discursos plurisótopos, ambivalentes, ricos en matices y con pluralidad de lecturas: los discursos artísticos. En el caso (III), el discurso promueve la multiplicación de los núcleos temáticos, presentados bajo un solo clasema: el discurso informativo es un ejemplo típico. El cuarto caso (IV) da origen a los discursos de vanguardia, del absurdo, experimentales, surrealistas, dadaístas, escritura automática, etcétera. Un solo verso de Vallejo lo ilustra de maravilla: "La paz, la avispa, el taco, las vertientes...".
La isotopía, entonces, es cada una de las líneas de lectura que impone el texto. Cuando son varias, como en los casos II, III y IV, la instancia enunciativa tiene que correlacionarlas entre sí, jerarquizarlas, homologarlas, homogeneizarlas. Tiene, además, que asignarles un modo de existencia en el discurso: realizado, virtualizado, actualizado, potencializado.

\section{La instancia de discurso}

La instancia de discurso designa el conjunto de operaciones, de operadores y de parámetros que controlan el discurso. Ese término genérico permite evitar la introducción prematura de la noción de sujeto. El acto es primero, y los componentes de su instancia son segundos, puesto que emergen del acto mismo.

Desde el punto de vista del discurso en acto, el acto es un acto de enunciación, que produce la función semiótica. Cuando se establece la fun- 
ción semiótica, la instancia de discurso opera un reparto entre el mundo exteroceptivo, que suministra los elementos del plano de la expresión, y el mundo interoceptivo, que suministra los elementos del plano del contenido. Ese reparto adquiere la forma de una toma de posición.

El primer acto es por lo tanto el de la "toma de posición": enunciando, la instancia de discurso enuncia su propia posición. Está dotada entonces de una presencia, que servirá de bito al conjunto de las demás operaciones. El operador de ese acto es el "cuerpo propio", un cuerpo sintiente y percibiente, que es la primera forma que adopta el actante de la enunciación. El "cuerpo propio" no es un cuerpo físico y biológico, de carne y hueso; es una categoría semiótica, que puede ser definida como "la forma significante de una experiencia sensible".

La toma de posición sensible está destinada a instalar una zona de referencia, estableciendo las dos grandes dimensiones de la sensibilidad perceptiva: la intensidad y la extensidad. En el caso de la intensidad, la toma de posición es una "mira" (en el sentido de "poner en la mira"); en el caso de la extensidad, la toma de posición es una "captación". La "mira" opera, entonces, en el ámbito de la intensidad: el "cuerpo propio" se torna hacia lo que suscita en él una fuerza sensible (percepti- va, afectiva). La captación opera, en cambio, en el ámbito de la extensión: el cuerpo propio percibe posiciones, distancias, dimensiones, cantidades.

Una vez cumplida la primera "toma de posición", ya puede funcionar la referencia: otras posiciones podrán ser reconocidas y puestas en relación con la primera. Y ese es el segundo acto fundador de la instancia de discurso: el desembrague realiza el paso de la posición original a otra posición. El desembrague es de orientación disjuntiva. Gracias a esa operación, el mundo del discurso se distingue de la simple "vivencia" inefable de la pura presencia. El discurso pierde ahí en intensidad, pero gana en extensión: nuevos espacios, nuevos momentos pueden ser explotados, y otros actantes pueden ser puestos en escena. El desembrague es, pues, por definición, pluralizante, y se presenta como un despliegue en extensión; pluraliza la instancia de discurso y su deixis restringida [yo-aquíahora]. El nuevo universo de discurso que es así abierto comporta, al menos virtualmente, una infinidad de espacios, de momentos y de actores.

Contra el desembrague se alza el embrague, que se esfuerza por retornar a la primera posición originaria. El embrague es de orientación conjuntiva; bajo su acción, la instancia de discurso trata de volver a encontrar la posición primera, aunque nunca podrá llegar a 


\section{Instancia de discurso}

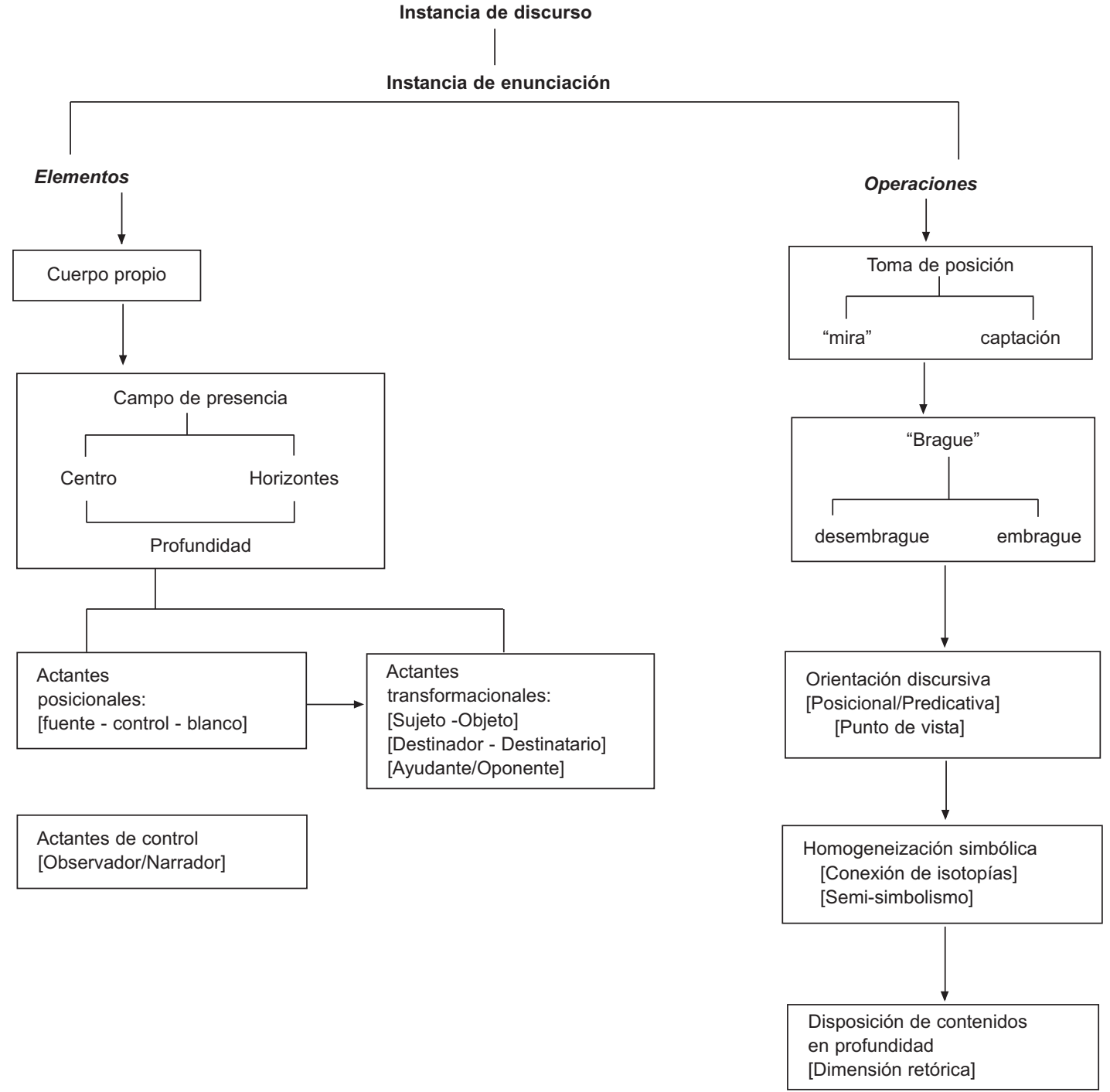


alcanzarla, porque el retorno a la posición original sería un retorno a lo inefable del "cuerpo propio", al simple presentimiento de la presencia. Pero puede al menos construir el simulacro. De esa forma, el discurso está en condiciones de proponer una representación simulada del momento (abora), del lugar (aqui) y de las personas de la enunciación $(Y O-T u ́)$. El embrague renuncia a la extensión, pues se acerca más al centro de referencia, y da prioridad a la intensidad: concentra de nuevo la instancia de discurso. El gé-nero poético es el resultado más patente de esa operación.

En el gesto mismo de retorno a la posición originaria (inaccesible), el discurso produce, al mismo tiempo, el simulacro de la deixis y el simulacro de una instancia única. La unicidad del sujeto de enunciación no es más que el efecto de sentido de un embrague bien forjado. En el verso de Vallejo: "Hay golpes en la vida, tan fuertes, yo no sé", la impresión de que el "yo" del poema es el "yo" de Vallejo es una mera ilusión; es el efecto de sentido que produce la operación del embrague; es un perfecto simulacro: ese "yo" es un "personaje" del poema, y no Vallejo. Así de simple. La situación ordinaria de la instancia de discurso es la pluralidad: pluralidad de roles, pluralidad de posiciones, pluralidad de tiempos, pluralidad de voces.

\section{La sintaxis del discurso}

La armadura general de la sintaxis del discurso, en la perspectiva de la presencia, es suministrada por los esquemas de tensión, puestos en secuencia y transformados eventualmente en esquemas canónicos. Del conjunto de las propiedades del discurso en acto, de la instancia de discurso y del campo posicional, esos diversos esquemas explotan en lo esencial las propiedades de la presencia, de la intensidad y de la extensidad.

Pero la sintaxis del discurso obedece a otras reglas, que explotan otras propiedades del discurso en acto. Hay que destacar, entre ellas: (1) la orientación discursiva, que impone en el campo de presencia la posición de las fuentes y de los blancos; (2) la bomogeneidad simbólica que procura el "cuerpo propio", puesto que él reúne y permite que se comuniquen ente sí la interoceptividad y la exteroceptividad; (3) la profundidad del campo posicional, que permite hacer coexistir y poner en perspectiva diversas "capas" de significación.

Respecto a la orientación discursi$v a$, el principio organizador es el punto de vista. En relación con la homogeneidad de los universos figurativos del discurso, el principio organizador es el semi-simbolismo, y más generalmente, todas las formas de conexión entre isotopias. En fin, respecto a la estratifica- 
ción en profundidad de las "capas" y dimensiones del discurso, el principio organizador es la retórica.

1. El punto de vista es una modalidad de la construcción del sentido. A ese respecto, cada punto de vista se organiza en torno a una instancia; la coexistencia de muchos puntos de vista en el discurso supone, pues, a la vez, que a cada punto de vista corresponde un campo posicional propio, y que el conjunto de esos campos posicionales son compatibles, de una manera o de otra, al interior del campo global del discurso.

El punto de vista se basa en el desajuste entre la mira y la captación, desajuste producido por la intervención del actante de control: alguna cosa que se opone a que la "captación" coincida con la "mira".

Pero el punto de vista es también el medio por el cual se busca optimizar esa "captación" imperfecta, es decir, adaptar la "captación" a lo que está "puesto en la mira".
Generalmente, la "mira" exige más de lo que la "captación" puede suministrar, y la "captación" tiende a alcanzar lo que la "mira" exige, y a ajustarse a ella.

La optimización es el acto propio del punto de vista: se disminuyen un poco las pretensiones de la "mira" y se mejora la "captación" para hacerlas congruentes. El punto de vista redefine permanentemente los límites del campo posicional. Ésa es otra de las propiedades del punto de vista: convertir un obstáculo en horizonte del campo, esto es, admitir el carácter limitado y particular de la percepción en acto, reconocer como irreductible la tensión entre la mira y la captación, y convertirla en fuente de la significación. El sentido emerge de esa tensión.

Globalmente, se pueden considerar cuatro grandes tipos de estrategias del punto de vista, sea actuando sobre la intensidad de la mira, sea actuando sobre la extensión de la captación, sea actuando sobre las dos dimensiones:

\begin{tabular}{|c|c|c|}
\cline { 2 - 3 } \multicolumn{1}{c|}{} & Mira intensa & Mira débil \\
\hline $\begin{array}{c}\text { Captación } \\
\text { extensa }\end{array}$ & $\begin{array}{c}\text { Estrategia } \\
\text { englobante }\end{array}$ & $\begin{array}{c}\text { Estrategia } \\
\text { acumulativa }\end{array}$ \\
\hline $\begin{array}{c}\text { Captación } \\
\text { restringida }\end{array}$ & $\begin{array}{c}\text { Estrategia } \\
\text { electiva }\end{array}$ & $\begin{array}{c}\text { Estrategia } \\
\text { particularizante }\end{array}$ \\
\hline
\end{tabular}


En el primer caso, el punto de vista será dominante o englobante; en el segundo caso, el punto de vista será acumulativo (incluso exhaustivo); en el tercer caso, el punto de vista será electivo (o también exclusivo); en el cuarto caso, el punto de vista será particular (o específico).

En cada tipo de punto de vista, el sentido atribuido al objeto se basa, en cada caso, en una morfología diferente: el objeto puede ser representado por una de sus partes (electivo), recompuesto por adición (acumulativo), captado de golpe como un todo (englobante), o reducido a un fragmento aislable (particular).

2. La cuestión de la conexión entre las diferentes isotopías se basa en el principio de la homogeneidad de todo universo semiótico, homogeneidad requerida entre el plano de la expresión y el plano del contenido. La conexión entre las isotopías puede ser asegurada por simples figuras que les son comunes: el rasgo de /altura/, por ejemplo, puede ser común, en el mismo discurso, a la isotopía de lo "celeste" y de lo "sagrado". Pero ese tipo de conexión, basada sobre una parte común puede ser simplemente un índice de coherencia. La homogeneidad solo estará asegurada si varios elementos de una isotopía en- tran en equivalencia con varios elementos de otra isotopía. La coherencia discursiva solo se logra si la conexión es establecida entre sistemas de valores y no entre términos aislados. Las conexiones establecidas término a término son de orden simbólico, en el sentido corriente de la palabra: la rosa "simboliza" el amor, el cielo "simboliza" lo divino, la balanza "simboliza" la justicia. Esas conexiones simbólicas son de escaso valor heurístico, porque o bien son tan convencionales que no aportan nada nuevo al discurso, o bien son fruto de las proyecciones personales del analista, y escapan por completo a cualquier tipo de racionalidad discursiva.

En cambio, las conexiones entre sistemas de valores particulares (entre oposiciones pertinentes) son el fruto de la praxis enunciativa, y concurren a la coherencia discursiva, construyendo los sistemas de valores del conjunto del discurso. Ese tipo de conexiones se denomina sistema semisimbólico.

El principio de los sistemas semisimbólicos fue establecido por Cl. Lévi-Strauss cuando planteó la fórmula del mito: la oposición entre dos figuras fue puesta en relación con la oposición entre dos funciones. La fórmula fue recogida por A. J. Greimas, quien la generalizó, reformulándola con mayor preci- 
sión: se produce un sistema semisimbólico cuando a una categoría del plano de la expresión $\left(s_{1} / s_{2}\right)$ corresponde una categoría del plano del contenido $\left(\mathrm{C}_{1} / \mathrm{C}_{2}\right)$ generando una correlación de homologación:

$\begin{array}{lll}\text { PLANO DE LA } & & \text { PLANO DEL } \\ \text { EXPRESIÓN } & & \text { CONTENIDO } \\ \boldsymbol{s}_{\mathbf{1}}: \boldsymbol{s}_{\mathbf{2}} & :: & \left(\boldsymbol{c}_{\mathbf{1}}: \boldsymbol{c}_{\mathbf{2}}\right)\end{array}$

Posteriormente, J.-M. Floch la convirtió en el instrumento principal de análisis de la imagen.

El ejemplo, ya mencionado, de los colores y el estado de la fruta ilustra claramente el funcionamiento del sistema:

$\begin{array}{lll}\text { PLANO DE LA } & & \text { PLANO DEL } \\ \text { EXPRESIÓN } & & \text { CONTENIDO } \\ \text { verde }: \text { dorado } & :: & \text { agraz }: \text { maduro }\end{array}$

Si el "cuerpo propio" percibiente se desplaza en el campo posicional y adopta otro punto de vista, podemos obtener la correlación siguiente:

\section{PLANO DE LA PLANO DEL EXPRESIÓN CONTENIDO agraz : maduro :: primavera : otoño}

Y con un nuevo desplazamiento:

\begin{tabular}{|c|c|}
\hline PLANO DE LA & PLANO DEL \\
\hline EXPRESIÓN & CONTENIDO \\
\hline
\end{tabular}

Como estos sistemas semisimbólicos son transitivos, se obtiene, para terminar, una correlación como la siguiente:

\section{PLANO DE LA PLANO DEL EXPRESIÓN CONTENIDO vede : dorado : : juventud : edad madura}

correlación que se encuentra con frecuencia en textos pictóricos y cinematográficos.

Si un conjunto de elementos puede ser puesto en relación con varios otros conjuntos, cambiará de forma con cada nueva asociación. El color puede ser puesto en relación con la madurez, con la emoción, con la circulación de automóviles (semáforo), etcétera. Por tanto, esos diferentes conjuntos se pueden superponer entre sí: con cada nueva correlación se genera un nuevo sistema semisimbólico.

En ese sentido, el dispositivo del sistema semi-simbólico es particularmente creativo, y al contrario de lo que sucede con el chato simbolismo, puede ser renovado en cada discurso por la instancia enunciativa. En el cine, particularmente en el cine expresionista y en el "cine negro”, se hizo clásico el sistema semisimbólico siguiente:

blanco : negro :: inocencia : maldad 
Pues bien; S. M. Eisenstein, en Alexandre Nevski, invirtió creativamente la correlación, obteniendo un fuerte efecto dramático y estético:

\section{blanco : negro :: maldad : bondad (teutones) (nacionales) (invasores) (defensores)}

La conexión semisimbólica entre isotopías puede ser establecida entre categorías próximas o distantes: cuanto más grande sea la distancia, más asegurada estará la homogeneidad global del discurso.

3. La coexistencia de diferentes isotopías en una misma zona del discurso supone que están todas ellas afectadas por grados de presencia diferentes, es decir, que son consideradas como más o menos intensas y como más o menos distantes de la posición de referencia del discurso.

Se trata ahora de la presencia de los contenidos mismos del discurso, presencia más o menos sentida y más o menos asumida por la instancia de enunciación. El campo posicional del discurso se convierte en un campo donde las isotopías están dispuestas en profundidad, en capas sucesivas, desde las más fuertemente presentes, en el centro del campo, hasta las más débilmente presentes, en la periferia.

Esa gradación de la presencia está bajo el control de la instancia de enunciación: cada capa está colocada bajo una mira más o menos intensa, o es captada como más o menos próxima o lejana. Dicho control enunciativo se ejerce en dos direcciones: la de la asunción, en términos de intensidad (sensible, afectiva), y la del despliegue, en términos de distancia (espacio-temporal, cognitiva). Las diferentes isotopías dispuestas en capas de profundidad discursiva son más o menos asumidas, y más o menos desplegadas: la instancia de discurso les impone o les retira su fuerza de enunciación (llamada a veces fuerza ilocutoria), las hace retroceder o avanzar en profundidad.

En ese dispositivo se ejerce la retórica. Y los "pequeños acontecimientos de conexión", hechos de conexiones y de tensiones locales entre isotopías conectadas entre sí, en los que se juegan diferentes modali-dades de coexistencia entre dichas isotopías, son tropos y figuras de retórica.

Se trata de los modos de existencia de los contenidos del discurso, determinados por los grados de su asunción y de su despliegue, atribuidos a cada contenido por la instancia de discurso. En tal sentido, es posible hacer corresponder a cada uno de los modos de presencia un modo de existencia de los contenidos discursivos: 


\begin{tabular}{|c|c|c|}
\cline { 2 - 3 } \multicolumn{1}{c|}{} & Mira intensa & Mira débil \\
\hline $\begin{array}{c}\text { Despliegue } \\
\text { extenso }\end{array}$ & $\begin{array}{c}\text { Plenitud } \\
\text { MODO REALIZADO }\end{array}$ & $\begin{array}{c}\text { Inanidad } \\
\text { MODO POTENCIALIZADO }\end{array}$ \\
\hline $\begin{array}{c}\text { Despliegue } \\
\text { restringido }\end{array}$ & $\begin{array}{c}\text { Carencia } \\
\text { MODO ACTUALIZADO }\end{array}$ & $\begin{array}{c}\text { Vacuidad } \\
\text { MODO VIRTUALIZADO }\end{array}$ \\
\hline
\end{tabular}

Toda figura retórica obedece a ese principio de base desde el momento en que asocia dos planos de enunciación distintos y asumidos de modo diferente. La metáfora y la metonimia invitan a superar el contenido directamente expresado y a asociar allí otro contenido, más general o perteneciente a otra isotopía; juegan, pues, con la disposición de los contenidos discursivos en profundidad y con modos de existencia diferentes.

En los versos de García Lorca:

Con el aire se batían las espadas de los lirios

Las operaciones de asunción y de despliegue, así como el modo de existencia atribuido a las isotopías en juego, se puede explicar de la siguiente manera:

La isotopía figurante es aquí la isotopía del movimiento de las espadas cuando se baten en duelo; la isotopía figurada es el roce de las hojas de los lirios por efecto del aire. La operación discursiva hace un quiebre entre la isotopía / mineral de las espadas y la isotopía /vegetal/ de las hojas. En un primer momento, la isotopía mineral de las espadas es puesta en el centro del campo de presencia, pero su asunción discursiva es débil, desde el momento en que el enunciado empieza con la expresión "Con el aire", que nos indica que la mira se ha puesto en otra parte, puesto que, de acuerdo con la experiencia perceptiva, las espadas no "se baten" con el aire. Y de pronto, y sin previo aviso, la isotopía se interrumpe, saltando inesperadamente al centro del campo el roce de las hojas de los lirios, fuertemente asumido por la instancia de discurso.

El contenido del batir de las espadas se encuentra realizado en un primer momento, pero queda virtualizado con la ruptura de la isotopía; mientras que el contenido del roce de las hojas de los lirios, que permanecía completamente potencializado, es de pronto reali- 
zado y asumido. Es evidente que el quiebre de isotopía se produce en base a una analogía perceptiva entre la forma visual de las hojas y la de las espadas, así como a una más lejana analogía entre el rumor de las hojas al rozarse y el ruido de las espadas al batirse.

Toda figura retórica constituye una microsecuencia discursiva, que comprende al menos una fase de "puesta en presencia" (por ejemplo, un conflicto entre dos enunciados o dos isotopías) y una fase de interpretación (por ejemplo, la resolución del conflicto por una analogía). En otros términos, cada figura podrá ser definida, al mismo tiempo, por el tipo de conexión (puesta en presencia) y por el tipo de resolución que requiere. La metáfora es una figura de conflicto semántico que se resuelve por analogía; la metonimia es una figura de conexión semántica que se resuelve por traslado de roles actanciales.

\section{Aportes de la semiótica}

La semiótica ha hecho importantes aportes a las ciencias humanas. Nos ha enseñado a ver de otra manera el mundo, desvaneciendo la visión ingenua a la que estábamos acostumbrados. Eso es evidente en campos como los de la literatura y el arte, pero también en los ámbitos de la historia y de la antropo- logía, de la política y del derecho, de la psicología y de la economía.

\section{Axiología/ideología}

Los objetos que buscan o rehúyen los sujetos son siempre objetos-de-valor. Pero resulta que los objetos del mundo, de por sí, son neutros. Solamente adquieren valor en el discurso cuando la instancia de enunciación los inviste con la categoría tímica, que organiza la dimensión propioceptiva del afecto en euforia/disforia. En ese momento, los objetos descriptivos, neutros, se convierten en valores, es decir, son axiologizados. La praxis enunciativa valoriza de manera diferente los mismos objetos neutros, proponiendo de esta suerte axiologías diferentes según las sociedades, los grupos y los individuos incluso. Por ejemplo, un individuo recibe una carta en un sobre con estampillas, lee la carta y rompe el sobre con las estampillas, mientras que su vecino, al ver lo que ha hecho, se lamenta del estropicio del que han sido objeto las estampillas. En el discurso del comportamiento del primero, las estampillas no han sido axiologizadas o lo han sido disfóricamente; en el del segundo, sí: porque es coleccionista. Si del ámbito individual pasamos al grupal, podemos observar cómo se axiologizan objetos como "riqueza" y "pobreza”. Para verlo con más claridad, será conveniente colocar esos valores en las posiciones que ofrece el cuadrado semiótico: 


\section{Discurso eclesiástico}

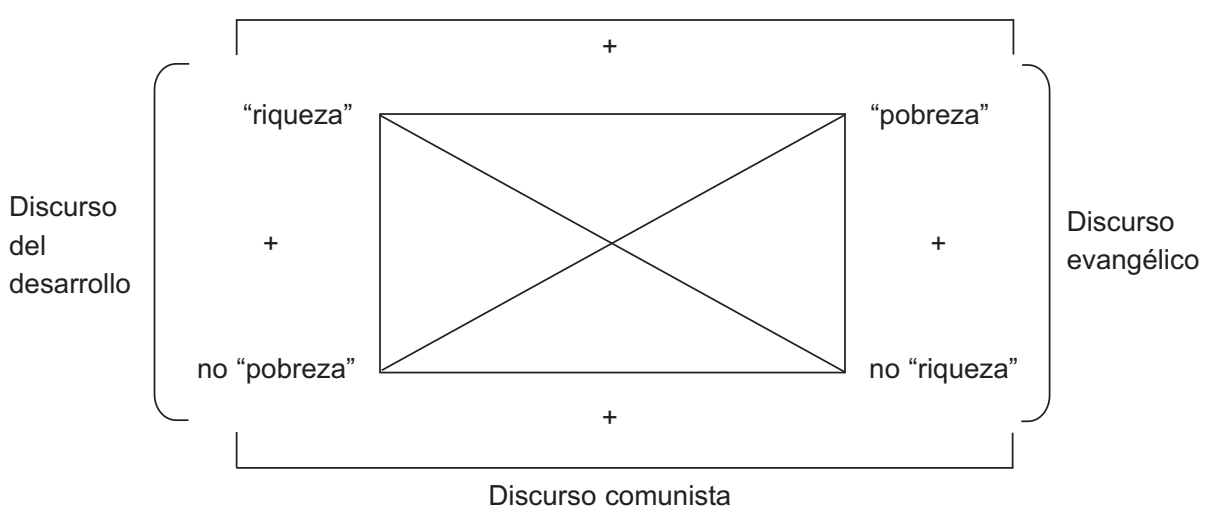

El discurso del desarrollo axiologiza eufóricamente la "riqueza sin pobreza"; el discurso evangélico euforiza la "pobreza sin riqueza"; el discurso comunista valoriza "la no riqueza y la no pobreza" (ni pobres ni ricos); el discurso eclesiástico trata de quedar bien con Dios y con el diablo, y, ju- gando con una doble isotopía, valora la "riqueza" (material) junto con la "pobreza" (de espíritu).

En el campo de la sociedad de consumo, J.-M. Floch ${ }^{1}$ elaboró un modelo axiológico que da cuenta de las distintas posiciones valorativas que pueden asumir los consumidores:

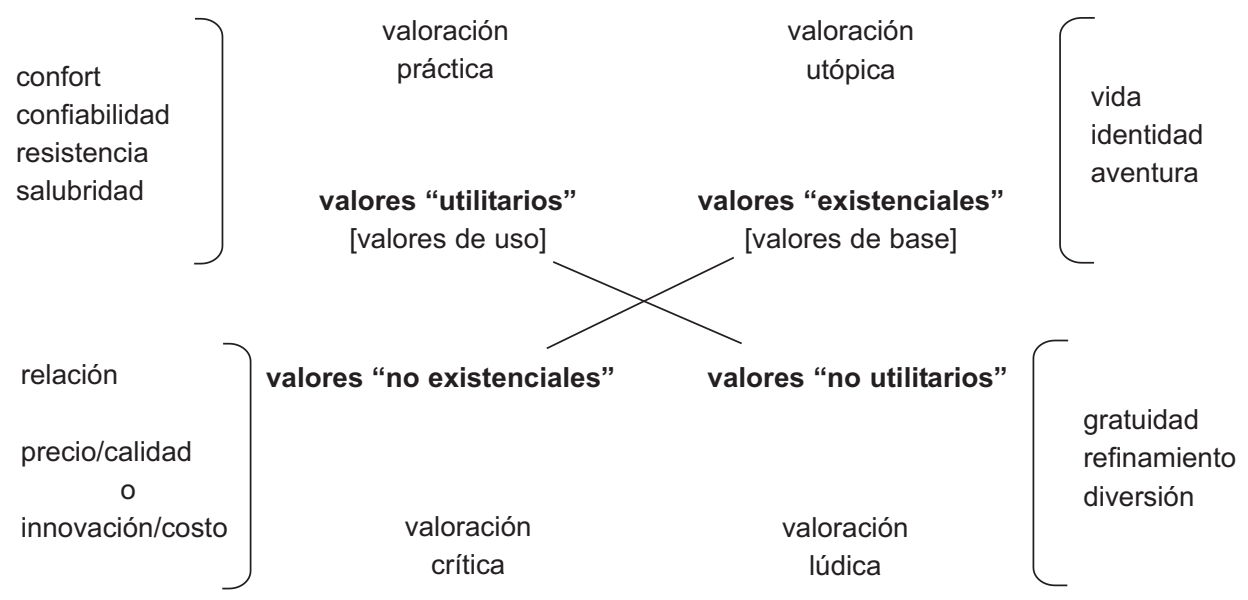

1 FLOCH, J.-M. Semiótica, marketing y comunicación. Barcelona: Paidós, 1992, p. 148. 
El modelo de Floch, elaborado en base al discurso publicitario, permite explicar el comportamiento de los consumidores de una sociedad como la nuestra.

El conjunto de valores así organizado constituye la axiología de una sociedad.

Pero los valores no permanecen estáticos sino que circulan en la sociedad, y al hacerlo, pasan de unos sujetos a otros. En consecuencia, los discursos sociales se ven obligados a relacionar los objetos-de-valor con los sujetos que los desean. En esa sintagmatización reside precisamente la ideología del discurso. La ideología no es una acumulación de ideas, depositadas en alguna región celeste; la ideología es una gramática de producción de sentido [E. Verón]. Un texto genera una ideología con la manera en que junta o separa los sujetos con los objetosvalor. El discurso cuyo programa narrativo de base conduce a la separación (=disjunción) de los sujetos con el objeto "propiedad privada", genera la ideología comunista; el discurso cuyo programa narrativo de base conduce a la unión (=conjunción) de los sujetos con el objeto "propiedad privada", genera la ideología capitalista. En otro ámbito discursivo, el discurso cuyo programa narrativo de base conduce a la unión (=conjunción) de la Iglesia y del Estado, genera la ideología del Estado confesional; el discurso cuyo pro- grama narrativo de base conduce a la separación (=disjunción) de la Iglesia y del Estado, genera la ideología del Estado laico. Y así por el estilo. La ideología emerge, pues, de la sintaxis narrativa del discurso; tampoco es algo dado de antemano.

\section{Enunciación/ Enunciado}

Otro de los aportes fundamentales para el análisis del discurso consiste en la teoría de la enunciación, tanto si se la considera como algo acabado, como algo que puede inferirse del discurso realizado, como si se la considera en el movimiento mismo del discurso en acto, del discurso en devenir. En cualquier caso, la semiótica nos ha enseñado a separar claramente las instancias de la enunciación de las del enunciado, instancias que la teoría literaria, y la artística en general, se han empeñado tercamente en confundir. Ya Greimas, hace algunos años (1973) alertaba sobre este grave problema:

Toda la confusión surge del hecho de que el sujeto de la enunciación, que es un sujeto lógico, es considerado por los lingüistas, y sobre todo por los literatos y por los filósofos, como un sujeto ontológico. La confusión, sin embargo, se disuelve fácilmente; pues si yo, en carne y hueso, como ser existente, digo: La tierra es redonda, entonces dicen que Greimas es el sujeto de la enunciación del enunciado La tierra es redonda. Pero, lingüísticamente, postular la existencia de Greimas significa postular la existencia 
de un referente exterior al lenguaje. Eso es antisaussuriano, y al hacerlo, toda la semiótica se derrumba. Pues eso es como decir que existe una realidad extralingüística que podemos conocer con métodos lingüísticos. Y por consiguiente, la principal conquista de la semiótica queda abolida y regresamos a la psicología del autor, a la biografía. Y ya conocen ustedes las consecuencias de esas posiciones. (...) El sujeto de la enunciación jamás puede ser captado directamente, y todos los "yo" que podamos encontrar en el discurso enunciado no son sujetos de la enunciación verdadera; solo son simulacros. (...) Los diferentes "yo" que aparecen en el enunciado son ya "yo" hablados y no "yo" que hablan. Porque el "yo" de la enunciación permanece siempre oculto, es siempre sobreentendido. $^{2}$

La enunciación se presenta, pues, bajo dos estatutos diferentes: la enunciación enunciada (simulacro) y la enunciación implícita, efecto de sentido del enunciado. Según Greimas y Courtés, autores del Diccionario razonado de la teoría del lenguaje, la enunciación enunciada se produce cuando, por medio de la operación semiótica del desembrague los actantes de la enunciación (enunciador/ enunciatario) son proyectados en el enunciado, asignándoles competencias enunciativas: se les otorga la palabra, la capacidad de decisión y de elección, y las demás competencias de la instancia enunciativa.

La enunciación implícita también está contenida en el enunciado, pero como un efecto de sentido del mismo. No podría estar en otra parte, ya que lo único que tenemos ante nosotros es el enunciado, con su particular estructura semiótica. Y es esa particular estructura la que nos permite (re)construir la instancia de la enunciación como un efecto del enunciado.

La instancia de la enunciación es muy compleja, y por medio de diferentes desembragues, proyecta en el enunciado sus diferentes roles. Uno de ellos, y de los más importantes, es el Narrador. El narrador es un "personaje" (en términos rigurosos, un Actante) creado por el enunciador por medio del lenguaje. La presencia del narrador se aprecia de inmediato, pues el autor, el que Greimas identifica como "de carne y hueso", no puede encontrarse en el lugar y en el tiempo de los acontecimientos que narra. Incluso cuando narra en primera persona, uno es el que narra y otro el que hace. En consecuencia, el sujeto que ve, y que viendo narra, es un sujeto construido por el enunciador e instalado en el discurso por desembrague. Alguna vez

2 GREIMAS, A. J. En torno al sentido. Madrid: Gredos, 1973. 
señaló M. Vargas Llosa que el "personaje" más difícil de construir en un relato es el narrador.

Como vemos, ni el narrador ni el enunciador se pueden confundir con el "autor de carne y hueso". Desde esa perspectiva, resulta verdaderamente pueril aquello de que la "tristeza" de Vallejo (el de carne y hueso) determina la tristeza (poética) de sus poemas, o aquello otro de que "Me moriré en París con aguacero" es una premonición de la muerte biológica del poeta en una tarde lluviosa de París. Y otras lindezas por el estilo.

Desde la perspectiva del discurso en acto, la instancia de enunciación es, como hemos dicho, el conjunto de operaciones, de operadores y de parámetros que controlan el discurso. El acto es un acto de enunciación que produce la función semiótica.

\section{Recuperación de la retórica}

La noción de isotopía y sus dispositivos operativos arrojan nueva luz sobre los procedimientos retóricos. Los dos grandes campos de la retórica están constituidos por la metáfora y por la metonimia. La metáfora trabaja por condensación; la metonimia, por desplazamiento.
Cuando, en un discurso cualquiera, se trunca la línea isotópica clasemática se producen efectos de sentido que van desde el chiste y el juego de palabras hasta la metáfora. La ruptura de la isotopía constituye una operación de sentido que genera la irrupción de lo inesperado en el discurso. Cuando esa irrupción degrada el nivel isotópico del sentido, se produce el chiste. Así por ejemplo:

- Vecino, lo siento; pero mi gata ha matado a tu perro.

- No puede ser, hermano; mi perro es un dóberman.

- Sí, pero mi gata es hidráulica.

La línea isotópica iniciada por "gata animal" se trunca inesperadamente para ser sustituida por el semema "gata mecánica”. La sustitución, no obstante, no es completa, pues la segunda "gata" conserva en la memoria la primera; por eso surge el chiste, precisamente. Se ha producido una condensación de las dos isotopías, pero la irrupción inesperada de la segunda se ha impuesto sobre la primera, sin anularla por completo. La instancia de discurso ha actualizado la segunda y potencializado la primera.

En un afiche publicitario de Luz del Sur se lee: 
Todos los días

de 6:30 a 10 de la

noche

abusan de ella;

$y$ en tu propia casa.

\section{Fotografía}

en la que se

aprecian rayos

$y$ haces luminosos.

Este abuso puede oscurecer tus días.

No desperdicies luz, especialmente entre las 6:30 y las 10 de la noche

La isotopía que dirige la lectura del texto comienza apoyándose en el clasema sexual: el abuso del que se habla conduce a pensar en un "abuso sexual", puesto que en ese contexto el pronombre "ella" es, salvo indicación contraria, un pronombre personal; por tanto, el abuso es un abuso de persona, y de persona femenina. Con el agravante de que el abuso se produce en la propia casa del enunciatario.

Pero en ese momento, la imagen se encarga de truncar la isotopía sexual, introduciendo el clasema/energético/ y con él una nueva isotopía, que se impone igualmente sobre la primera. Este juego de palabras es muy utilizado en el discurso publicitario.

En los dos casos comentados, podemos observar que la línea de lectura (=isotopía), de un nivel, considerado superior, /animal/ en el primer ejemplo; /personal/ (=/humano/), en el segun- do, pasa a un nivel considerado inferior, /mecánico/ en el primer caso; /energético/, en el segundo. Graficando:

I. Isotopía $1^{\text {a }}$. /animal/

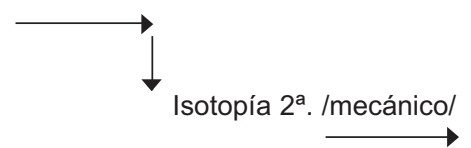

II. Isotopía $1^{\text {a }}$. /personal/

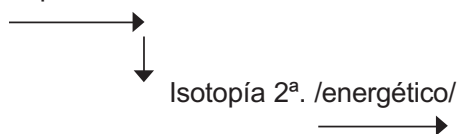

En la metáfora, en cambio, la ruptura de isotopía se produce generalmente hacia arriba:

\section{La luna vino a la fragua con su polisón de nardos.}

\section{F. García Lorca}

En este caso, la condensación es completa; casi no nos permite imaginar la isotopía que ha sido sustituida, y el enunciado nos ofrece solamente la isotopía sustituyente. La luna se nos presenta ataviada con vestidos de mujer: el polisón o miriñaque adornado de flores. La fuerza expresiva de la metáfora consiste precisamente en eso: en crear una condensación total entre los clasemas /astronómico/ y /vestimentario/, que arrastra tras de sí el clasema más genérico/femenino/. La luna, destacando en la bóveda celeste, 
tachonada de estrellas, se convierte de golpe en una dama con polisón de nardos. En este caso, la segunda isotopía es más noble que la primera, y trasunta la materia original en una figura elegante:

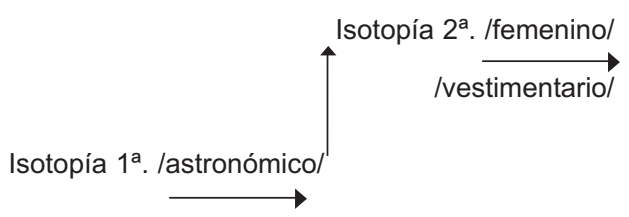

Sin embargo, por la condensación producida, las dos isotopías trabajan juntas y enriquecen la densidad de la significación, con los efectos estéticos correspondientes.

La metonimia, en cambio, se acoge al esquema discursivo (III), según el cual, la isotopía se apoya en la redundancia de los semas nucleares. Por la yuxtaposición de dos núcleos sémicos, se produce un desplazamiento de sentido entre uno y otro, manteniendo el mismo clasema organizador del contexto.

En el socorrido ejemplo de metonimia:

\section{- Te invito a tomar una copa,}

la contigüidad existente entre el núcleo sémico de "copa" (/continente/) y el núcleo sémico de "vino" (/contenido/) permite desplazar el rol actancial /objeto/ de "vino" a "copa" y de "copa" a "vino". El /objeto/ "copa" es actualizado pero no asumido por el enunciador, mientras que el /objeto/ "vino" es asumido aunque está potencializado; el /objeto/ "vino" es actualizado y asumido por el enunciatario, mientras que mantiene al /objeto/ "copa" en estado virtualizado.

En un spot publicitario de la firma Pfizer, aparecen tres encuadres sucesivos: en el primero vemos en primer término unas cuerdas o barras que cruzan la pantalla de lado a lado, horizontalmente; y empezamos a escuchar un tintineo metálico particular en off, sobre un fondo difuminado, en el que se mueven unas masas informes. En el segundo encuadre, se aprecia la cabecera de una cama, de la cual cuelga una correa cuya hebilla golpea suavemente sobre el catre, produciendo, ahora en in, el sonido que antes percibíamos, mientras que al fondo, las masas amorfas siguen moviéndose. En el tercero, aparece una joven parejaterminando de hacer el amor, con sonrisas de satisfacción. La hebilla ya no se oye. Pfizer es la firma que fabrica el producto viagra, que favorece la función eréctil y por tanto proporciona un acto sexual satisfactorio. Modelo, sin duda, de metonimia visual. Por la contigüidad entre los núcleos sémicos de "correa" y "cuerpo humano", el sentido se desplaza de unos a otros, sin abandonar el clasema/sexual/. Desde el 
punto de vista de la profundidad existencial, el movimiento y el sonido de la hebilla están realizados en la puesta en escena, pero no son asumidos por la instancia de discurso, pues la "mira" está puesta en el movimiento y sonido de los cuerpos, los cuales, a su vez, se encuentran actualizados y asumidos como causa del movimiento de la hebilla. En un avance más de la yuxtaposición, la plenitud de felicidad, realizada en los rostros sonrientes de la pareja de la última secuencia, es asumida por el enunciatario, destinatario final del mensaje publicitario. El desplazamiento de sentido se apoya igualmente aquí en el traslado de roles actanciales: el movimiento del cuerpo se traslada a un accesorio del cuerpo.

Como en el primer caso, la energía de sentido corre de lo /material inerte/ [hebilla] a lo /corporal/[acto sexual] para terminar en lo /espiritual/ [satisfacción]. El juego semiótico ha sido consumado.

En todos los casos, la fuerza de la asunción y el modo de existencia que la instancia de enunciación [enunciador/enunciatario] otorga a las entidades semióticas, determina la morfología de la estructura de significación.

\section{Diálogo entre culturas}

A partir de los estudios de I. Lotman sobre la semiosfera, J. Fontanille (2000) ha elaborado un modelo semiótico que permite explicar el diálogo entre las culturas. El porvenir de un aporte exterior que ingresa dentro de las fronteras de una cultura establecida, puede seguir los siguientes recorridos:

- El aporte exterior es percibido como brillante y singular. En consecuencia, se beneficia de una axiología ambivalente: positiva en cuanto a la sorpresa o al interés que suscita, negativa en cuanto a su fuerza subversiva en relación con la cultura que lo acoge.

- El aporte exterior es imitado, reproducido y transpuesto en términos de lo "propio", de "lo nuestro", lo cual le permite ser difundido e integrado por entero en el campo interior, de suerte que pierde todo bri1lo, y en consecuencia, deja de ser sorprendente e inquietante.

- El aporte exterior no es reconocido como extraño; su origen es incluso discutido, se le retira todo lo que tenía de específico, se lo oculta para asimilarlo mejor a la cultura que lo ha acogido.

- El aporte exterior, irreconocible como tal, es erigido en norma universal, y es propuesto de retorno, no solamente en los límites del dominio interior sino también en los dominios exteriores, como parangón de toda cultura, como signo de la civilización por excelencia. 
La explotación que la Semiótica tensiva hace de esos recorridos se apoya en las propiedades de campo que pone en evidencia: los movimientos de actualización y de potencialización de las formas, en el recorrido que efectúan por la semiosfera, afectan principalmente la intensidad y la extensidad (cantidad) de su recepción y de su difusión.

El paradigma de las formas de diálogo entre campos semióticos conlleva las tensiones siguientes:

- tensiones entre la abertura del campo [casos $B$ y $D$ ] y el cierre del campo [casos $A$ y $C$ ].
- tensiones entre una intensidad afectiva fuerte (intensidad de percepción y de recepción) [casos $A$ y $D$ ] y una intensidad débil [casos $B$ y $C$ ];

- tensiones referidas a la extensión y a la cantidad, importante y en expansión en los casos $B$ y $D$, restringida y en concentración en los casos $A$ y $C$.

La praxis enunciativa juega en dos dimensiones esenciales: la intensidad y la cantidad. Su campo de ejercicio, la semiosfera, acoge los diferentes aportes y los transforma en cuatro fases, definidas con ayuda del esquema siguiente:

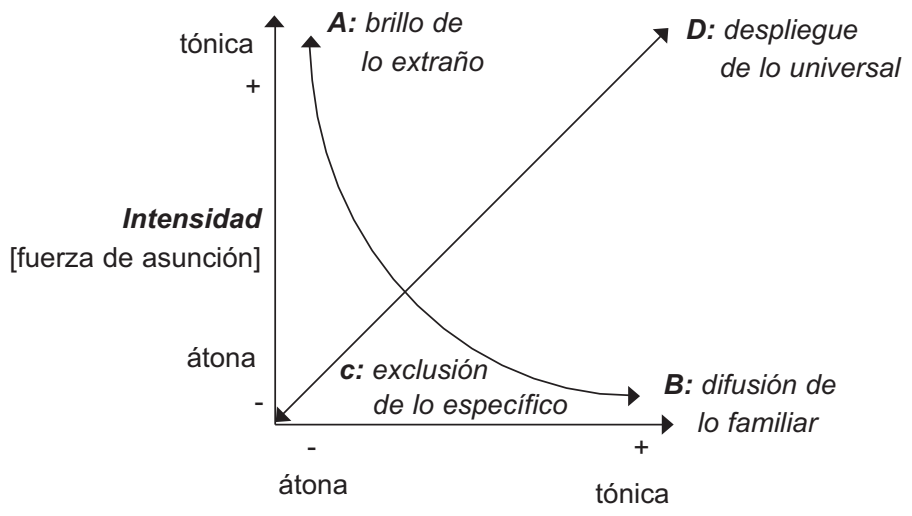

despliegue y difusión 
Un ejemplo típico del recorrido descrito por Lotman y modelado por Fontanille lo encontramos en el destino de esa forma de vida que conocemos como democracia, cuyos orígenes se remontan a la Carta Magna, suscrita por Juan sin Tierra. La noción de "democracia" penetra las fronteras de la cultura francesa:

- Los franceses la reciben como algo innovador y deslumbrante, aunque, por otro lado, la consideran como una amenaza para el sistema feudal instalado.

- Más tarde, con los humanistas, esa noción se va difundiendo entre la burguesía emergente. Durante la época de la Ilustración, ya se considera como una noción propia, y se va instalando en las mentes de los franceses, que no se sienten ya amenazados por sus propuestas.

- Con la Revolución Francesa, la democracia es considerada como algo generado en su propia cultura, su origen no es discutido, porque no tiene nada de extraño. Se convierte en el motor de la revolución.

- Finalmente, con la Declaración de los Derechos del Hombre y del Ciudadano, la cultura francesa propone a todas las naciones la forma de vida en democracia como un valor universal indiscutible.

\section{Bibliografía}

BLANCO, D. y R. BUENO. Metodología del análisis semiótico. Lima: Universidad de Lima, 1983, 1989. 1980.

FLOCH, J.-M. Semiótica, marketing y comunicación. Barcelona: Paidós., 1992. [Versión original: París, Presses Universitaires de France, 1990].

FONTANILlE, J. Semiótica del discurso. Lima: Universidad de Lima/ Fondo de Cultura Económica, 2001. [Versión original: Limoges, PULIM, 1998].

—. Sémiotique et littérature. Essais de méthode. París: Presses Universitaires de France, 1999.

GREIMAS, A. J. De la imperfección. México-Puebla: Fondo de Cultura Económica/UAP, 1990. [Edición original: Périgueux, P. Franlac, 1987].

—. Del sentido II. Ensayos semióticos. Madrid: Gredos, 1989. [Versión original: Du sens II. Essais sémiotiques. París, Éditions du Seuil, 1983].

- En torno al sentido, Madrid: Gredos, 1973. [Versión original: Du sens. Essais sémiotiques, París, Éditions du Seuil, 1970]. 
—. Semántica estructural. Investigación metodológica. Madrid: Gredos, 1971. [Versión original: París, Librairie Larousse, 1966].

GREIMAS, A. J. y J. COURTÉS. Sémiotique. Dictionnaire raisonné de la théorie du langage I. París: Hachette, 1986. [En español: Semiótica. Diccionario razonado de la teoría del lenguaje. Madrid: Gredos, tomo 1, 1982, tomo 2, 1991].

—. Sémiotique. Dictionnaire raisonné de la théorie du langage. París: Hachette, 1979.
GREIMAS, A. J. y J. FONTANILLE. Semiótica de las pasiones. MéxicoPuebla: Siglo XXI/UAP, 1994. [Edición original: París, Seuil, 1991].

QUEZADA, O. Semiótica generativa. Bases teóricas: Lima: Universidad de Lima, 1991.

ZILBERBERG, Cl. Ensayos sobre semiótica tensiva. Lima: Universidad de Lima/Fondo de Cultura Económica, 2000. 